\title{
Guillain-Barre Syndrome, CTCAE
}

National Cancer Institute

\section{Source}

National Cancer Institute. Guillain-Barre Syndrome, CT CAE. NCI Thesaurus. Code

C143231.

A disorder characterized by the body's immune system attacking the peripheral nervous system causing ascending paralysis. 